\title{
Arm Swing Identification Method with Template Update for Long Term Stability
}

\author{
Kenji Matsuo $^{1}$, Fuminori Okumura ${ }^{2}$, Masayuki Hashimoto ${ }^{1}$, \\ Shigeyuki Sakazawa ${ }^{1}$, and Yoshinori Hatori ${ }^{2}$ \\ ${ }^{1}$ KDDI R\&D Laboratories Inc., \\ Ohara 2-1-15, Fujimino-shi, Saitama, 356-8502 Japan \\ matsuo@kddilabs.jp \\ ${ }^{2}$ Tokyo Institute of Technology, \\ Nagatsuta 4259, Midori-ku, Yokohama-shi, 226-8502, Japan
}

\begin{abstract}
This paper proposes a novel method for biometric identification, based on arm swing motions with a template update in order to improve long term stability. In our previous work, we studied arm swing identification and proposed a basic method to realize a personal identification function on mobile terminals. The method compares the acceleration signals of arm swing motion as individual characteristics, with the tolerant similarity measurement between two arm swing motions via DP-matching, which enables users to unlock a mobile terminal simply by swinging it. However, the method has a problem with long term stability. In other words, the arm swing motions of identical individuals tend to fluctuate among every trial. Furthermore, the difference between the enrolled and trial motions increases over time. Therefore in this paper, we propose an update approach to the enrollment template for DPmatching to solve this problem. We employ an efficient adaptive update method using a minimum route determination algorithm in DP-matching. Identification experiments involving 12 persons over 6 weeks confirm the proposed method achieves a superior equal error rate of $4.0 \%$ than the conventional method, which has an equal error rate of $14.7 \%$.
\end{abstract}

Keywords: behavior identification, template update, arm swing motion, cellular phone security, DP-matching.

\section{Introduction}

Recent mobile terminals, such as cellular phones, come equipped with various functionalities and require flexible means of authentication based on the nature of each function. Processing electronic payments on the phone requires highly secure authentication, while simple phone call functionality does not necessarily require such strict security. Current implementations of such devices employ a PIN code and/or fingerprint authentication. Although both methods can achieve a relatively high security level, the PIN code may often be annoying for users to enter, while biometrics involves a high risk in the case of theft of the registered fingerprint data, which is an unchangeable personal feature. 
These days, human behaviors such as speech, signatures and gestures are employed for easy-to-use authentications [1]-[3]. As one of those research activities, we have proposed a new means of biometric identification, based on the similarity of arm swing motion [4]. Since arm swing motions are captured naturally through everyday behavior, they are suitable as a means of personal identification on mobile terminals, such as cellular phones. This method authenticates the genuine identity of individuals by comparing the acceleration signals acquired when they move their arms. The principles of differentiation are derived from the individual differences that exist in arm length, muscle strength, and the manner of swinging. Our method records arm swing motions on a serial basis and measures the similarity between two arm swing motions via DP-matching.

In general, behavior, including arm swing motion, is apt to fluctuate, since even identical individuals show slightly different behavior in every operation. Gaps from the enrollment template increase over time, since human muscles grow or decline and the swing pattern is forgotten unconsciously. Therefore, the behavior identifications need a template update to tolerate such long term fluctuations. Previously, the problems of long term fluctuations were studied for other biometrics identifications and Uludag et. al. proposed a fingerprint identification method using multiple templates to improve the discrimination accuracy [5]. They also proposed a template update procedure, in which a template set with discriminative characteristics is chosen from among several fingerprints acquired. However, this method requires computationally complex tasks to choose the best fingerprint, since similarity calculation between the new fingerprint data and all data in the templates, as well as the layered clustering calculation on a binary partition tree, is required. This method also requires considerable storage to hold the multiple templates. Kato et al. proposed an update method of an acoustic model for speech authentication using HMM [1]. The update method calculates the similarity for each trial and uses the new speech data for updating if the score exceeds a certain threshold. It also requires considerable storage for original speech data, in addition to the acoustic model, as well as vast computation for the reprocessing of models. Thus, it is difficult to perform these update processes on mobile terminals with limited CPU power and memory capacity.

In this paper, we propose a new approach to solve the fluctuations in arm swing identification occurring over time. The proposed method updates the enrollment template, using the minimum route of DP-matching to achieve long term stability. It does not require the original data used to create the template in previous operations, but merely requires the latest template and the new input data to update the template. Its performance was confirmed through identification experiments, carried out by 12 persons over a 6 week period.

\section{Arm Swing Identification}

Figure 1 shows the concept of the arm swing identification for cellular phones, which simplifies owner identification: This enables the owner to unlock his/her cellular phone by simply swinging it without any difficulty. 

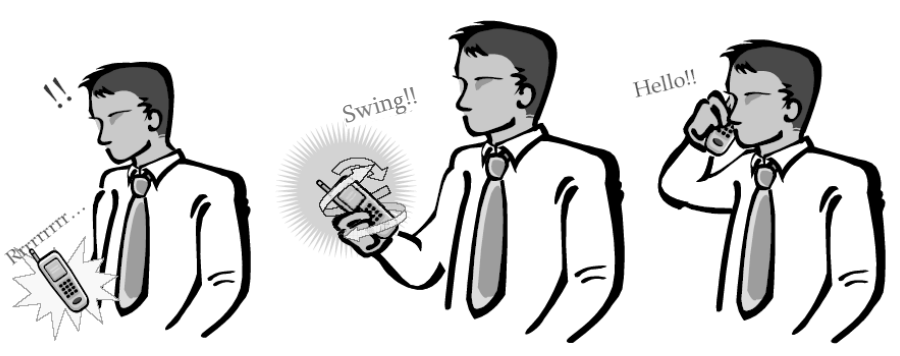

Fig. 1. Arm swing identification for cellular phone

However, it is difficult to acquire arm swing tracks precisely due to the accumulation of drift errors due to the noise of the motion sensor. Therefore, we proposed an identification method using an arm swing motion acquired by an acceleration sensor rather than a track [4]. The method represents the basic element of the method proposed in this paper and is thus described in detail in the following subsections.

\subsection{Extraction of Acceleration Signals During the Motion Interval}

All acceleration signals during the arm swing motion are obtained with a fixed sampling interval using an acceleration sensor. $\boldsymbol{a}[t]$ represents the 3 dimensional acceleration signals recorded at time $t$, while $a_{x}[t], a_{y}[t]$, and $a_{z}[t]$ are the $x, y$, and $z$ axis elements of $\boldsymbol{a}[t]$ respectively.

$$
\boldsymbol{a}[t]=\left(a_{x}[t], a_{y}[t], a_{z}[t]\right)
$$

The magnitude of acceleration signals $|\boldsymbol{a}[t]|$ is defined in the following equation:

$$
|\boldsymbol{a}[t]|=\sqrt{a_{x}[t]^{2}+a_{y}[t]^{2}+a_{z}[t]^{2}}
$$

Figure 2 shows the acceleration sensor and acceleration signals obtained when shaking up and down 5 times. The figure also shows the variance of $\mathrm{a}[\mathrm{t}]$ in certain past samples. Although considerable variance is evident during arm swinging, this

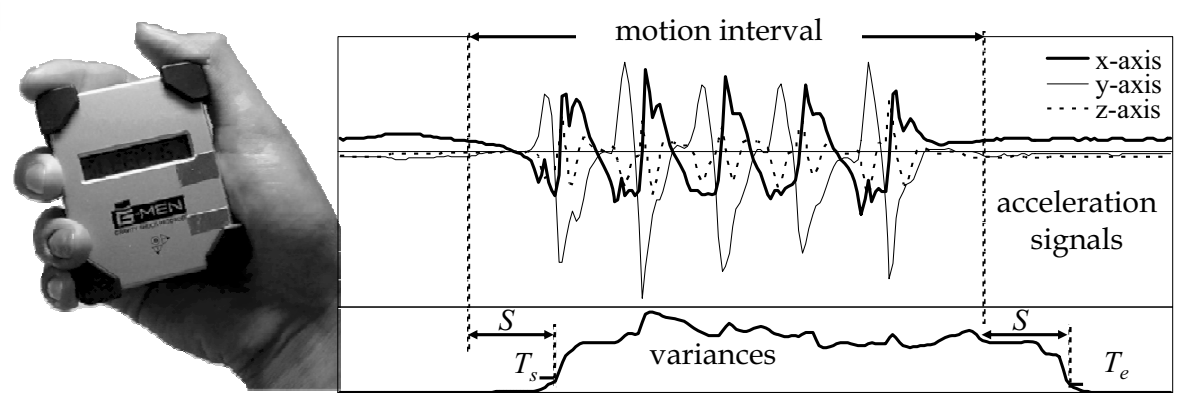

Fig. 2. Acceleration sensor and extraction of the motion interval 
diminishes when the arms are kept still. Therefore, we judge the start and end of the significant motion based on this variance: If the variance in the previous $S$ samples exceeds a threshold $T_{s}$, the starting point of $S$ samples is judged to be the start of the motion, while if the variance falls below a threshold $T_{e}$, the starting point of $S$ samples is judged to be the end of the motion. Extracted acceleration signals during the motion interval are recorded as the enrollment template $\boldsymbol{u}$ in the enrollment operation or input motion $v$ in identification trials respectively.

\subsection{Error Angle}

The difference between the dual arm swing motions $\boldsymbol{u}$ and $\boldsymbol{v}$ is calculated based on the sum of the error angles. $\boldsymbol{u}[i]$ is an acceleration vector in the enrollment template at the sampling time $i(i=1,2, \ldots, M)$, while $v[j]$ is an acceleration vector in the input motion at sampling time $j(j=1,2, \ldots, N)$. The error angle $\theta[i, j]$ is the inner angle between $\boldsymbol{u}[i]$ and $\boldsymbol{v}[j]$, as defined in the following:

$$
\begin{gathered}
\theta[i, j]=\arccos \frac{(\boldsymbol{u}[i], \boldsymbol{v}[j])}{|\boldsymbol{u}[i]||\boldsymbol{v}[j]|} \\
0 \leq \theta[i, j] \leq \pi
\end{gathered}
$$

The value comes up to 0 when the directions of both acceleration vectors are identical. Conversely, the error angle increases when the directions differ. Although arm strengths often cause the amplitude of the acceleration signal to vary with every trial, the error angle is independent of the amplitude; hence the latter need not be taken into account [4].

\subsection{DP-Matching Algorithm}

As previously mentioned, arm swing motions tend to fluctuate, even in identical individuals, in terms of the positional shift, partial expansion and contraction, because the arm swing speed varies for each trial. Therefore, when evaluating the similarity between the enrollment template $\boldsymbol{u}$ and input motion $\boldsymbol{v}$, we use the difference $D(\boldsymbol{u}, \boldsymbol{v})$ with DP-matching, which is a measurement method with a high tolerance for gaps caused by such fluctuations.

The difference $D(\boldsymbol{u}, \boldsymbol{v})$ is an accumulation of the error angles during the motion interval. The difference is defined by the following recursive equations:

$$
\begin{gathered}
D(\boldsymbol{u}, \boldsymbol{v})=\frac{D[M, N]}{M+N} \\
D[i, j]=\min \left\{\begin{array}{c}
D[i-1, j-1]+\theta[i, j], \\
D[i, j-1]+\theta[i, j]+P \\
D[i-1, j]+\theta[i, j]+P
\end{array}\right\} \\
D[0,0]=0, D[0,1]=D[1,0]=\infty
\end{gathered}
$$

$M$ and $N$ represent the length of the enrollment template $\boldsymbol{u}$ and the input motion $\boldsymbol{v}$ respectively. 
The algorithm is equivalent to searching for a minimum route, as shown in Figure 3, namely one where the difference among all the routes on the table is minimized. The indexes of the column and row correspond to the sampling times of $\boldsymbol{u}$ and $\boldsymbol{v}$ respectively, while the error angle $\theta[i, j]$ at points $[i, j]$ is measured from $\boldsymbol{u}[i]$ and $v[j]$, and $D(\boldsymbol{u}[i], \boldsymbol{v}[j])$ is calculated recursively. $P$ is a toll penalty, which is imposed if a time-shifted sample is chosen when the temporal difference $D[i, j]$ is calculated. This makes it hard to choose samples of different times, while $P$ is added when gaps are exceptionally permitted. The experiments mentioned later set $P=0.2$. An example of a minimum route determined by DP-matching is illustrated in the form of bold lined blocks.

The smaller the measured difference $D(\boldsymbol{u}, \boldsymbol{v})$, the more $\boldsymbol{u}$ and $\boldsymbol{v}$ resemble each other. Therefore, the threshold $T$ is introduced for personal identification. If $D(\boldsymbol{u}, \boldsymbol{v})$ falls below $T$, the person is accepted as a valid individual. Conversely, if $D(\boldsymbol{u}, \boldsymbol{v})$ exceeds $T$, the person is rejected.

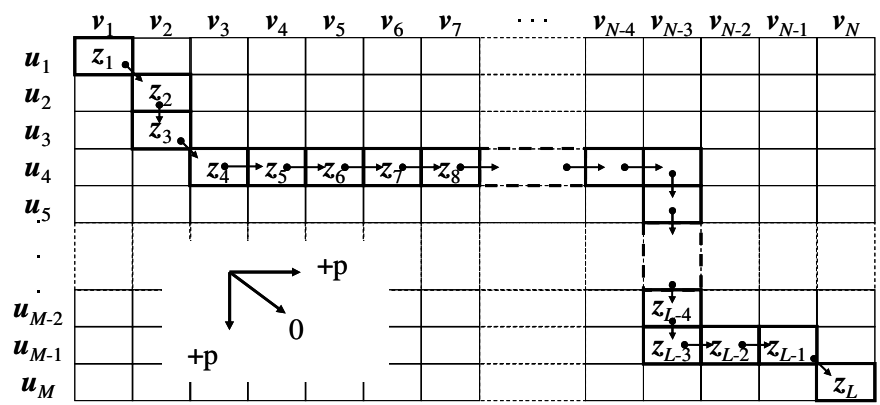

Fig. 3. DP-matching table. A minimum route is that illustrated by bold lined blocks.

\section{Proposed Method}

The basic arm swing authentication is relatively effective as reported in [4]. However the False Rejection Rate deteriorates over time, because the determination algorithm does not reflect the alteration of the arm swing. Therefore, this section proposes a method to update the enrollment template. The method does so using the minimum route, which is derived from DP-matching on the latest acceleration data determined as valid authentication. Figure 4 provides a conceptual explanation on the update process, using the distribution of templates in the arm swing motion space and its probability density for a person. $\boldsymbol{u}$ is the initial enrollment template, while the first and second updates create new templates, $\boldsymbol{u}_{1}$ and $\boldsymbol{u}_{2}$, respectively and the template becomes $\boldsymbol{u}_{\text {End }}$ finally. During each update process, the updated template approaches the center of the distribution.

\subsection{Template Update Using the Minimum Route in DP-Matching}

Most pairs of acceleration vectors on the minimum route usually have a small error angle and high similarity. However the similarity of certain pairs decreases over time, 

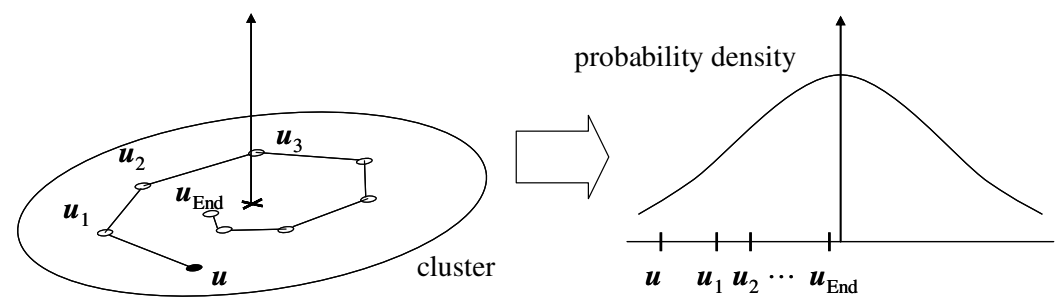

Fig. 4. Concept of the template update

even on the minimum route. The notation $z$ indicates the update template generated from the current enrollment template $\boldsymbol{u}$ and an input motion $\boldsymbol{v} . \boldsymbol{z}[k]$ at sampling time $k$ $(k=1,2, \ldots, L)$ in the updated template is calculated with the weighted average between the pair of acceleration vectors $\boldsymbol{u}[i]$ and $\boldsymbol{v}[j]$ on the minimum route, as defined in the following equations:

$$
\begin{gathered}
\boldsymbol{z}[k]=w_{u} \cdot \boldsymbol{u}[i]+w_{v} \cdot \boldsymbol{v}[j] \\
w_{u}+w_{v}=1
\end{gathered}
$$

Where, $w_{u}$ and $w_{v}$ are weights for $\boldsymbol{u}[i]$ and $\boldsymbol{v}$ [j]. For example, $\boldsymbol{z}$ [3] at sampling time $k=3$ comprises a weighted average between $\boldsymbol{u}$ [3] and $\boldsymbol{v}$ [2] in Figure 3. The weighted average has the advantages that the acceleration signal $z[k]$ in the updated template retains values close to $\boldsymbol{u}[i]$ in the enrollment template if $\boldsymbol{u}[i]$ and $\boldsymbol{v}[j]$ are similar. Conversely, $\boldsymbol{z}[k]$ adopts a new element, which exists not in $\boldsymbol{u}[i]$, in the current enrollment template, but rather in $v[j]$ in input motion, if $\boldsymbol{u}[i]$ and $\boldsymbol{v}[j]$ are dissimilar.

However, the above simple update method always expands the length $L$ of the updated motion series, because DP-matching inevitably finds the minimum route, where length $L$ has the following relations: $L \geq \max (M, N)$. Therefore, we employ a normalization process to avoid expanding the length $L$. The length of the updated template is fitted to the average length, as calculated through previously identified trials.

\subsection{Adaptive Update According to Adequacy Measurement}

The simple update method may suffer from over-training caused by accidental movement during the arm swing, even if the trial is determined to be valid. Besides, with computational complexity in mind, unnecessary update operations should be avoided. If the latest minimum route in DP-matching is relatively similar to previous ones, there is little improvement in the enrollment template. Consequently, the use of an adaptive update algorithm is necessary in order to ensure the enrollment template remains effective and to reduce the number of unnecessary updates.

Figure 5 shows the adaptive update algorithm, where the threshold $T$ defined in 2.3 is introduced for personal identification and two thresholds, $T_{l}$ and $T_{h}$, are set to judge the necessity of an update. If a difference $D(\boldsymbol{u}, \boldsymbol{v})$ exists between $T_{l}$ and $T_{h}$, the enrollment template is simply updated. The two thresholds $T_{l}$ and $T_{h}$ are determined from the following two criteria: 
- Upper threshold $T_{h}$ : If a considerable difference from the current enrollment template is apparent, such input motions might include many new elements, which are not contained in the current enrollment template. Renewal may actively incorporate those new elements into an updated template in this case. However, if the difference is excessive, input motions might include many accidental elements, which degrade the reliability of the updated template. Accordingly, the upper threshold $T_{h}$ is applied to prevent the accidental elements from being reflected on the updated template.

- Lower threshold $T_{l}$ : If the difference is very modest, such input motion is almost identical to the current enrollment template and there are no new elements which should be taken into account in the updated template. Therefore, the lower threshold $T_{l}$ is equipped to prevent the increase in needless updates.

Thresholds $T_{l}$ and $T_{h}$, having satisfied the above two criteria, can be determined based on the probability distribution of differences between the arm swing motions of identical individuals. Figure 6 shows an example of probability distribution actually generated from 7800 samples by 12 persons in a preliminary experiment. Both $\boldsymbol{u}$ and $\boldsymbol{v}$, used to calculate the difference $D$ in Figure 6, are the arm swing motions of identical individuals. The pairs of $T_{l}$ and $T_{h}$ in later experiments are calculated based on the probability intervals of the difference distribution, such as $95 \%, 90 \%$, and $80 \%$.

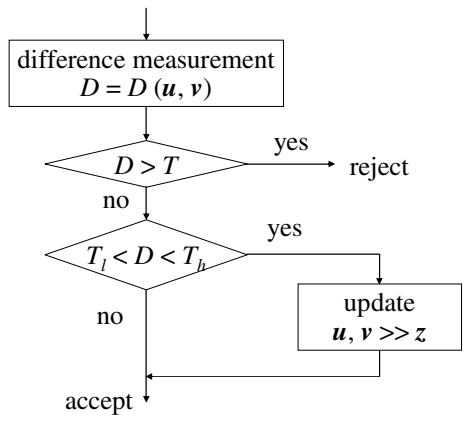

Fig. 5. Block diagram of adaptive update

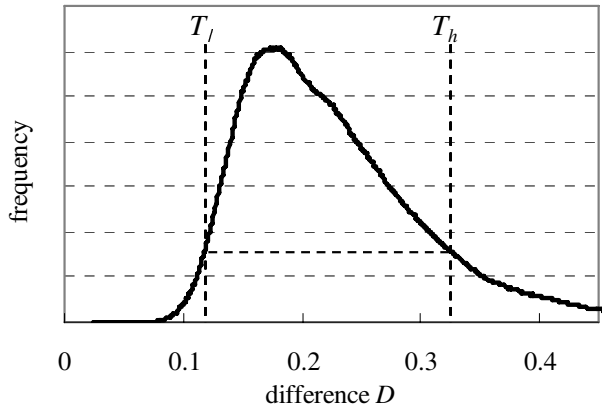

Fig. 6. Probability distribution of differences in identical people

\section{Experimental Results and Discussions}

Identification experiments involving 12 persons were conducted as personal verification for logging onto a PC. Each user swings his/her arm before using his/her own PC regularly and an acceleration sensor is connected to the PC via a USB cable. The sensor measures the acceleration signals with a range of $10 \mathrm{G}$ and sampling frequency of $100 \mathrm{~Hz}$. The extraction parameters $S=50, T_{s}=1.0$, and $T_{e}=0.2$ are set according to the results of preliminary experiments. A total of $814 \mathrm{arm}$ swing motions are accumulated over a 6 week period, as each individual tries 30 to 100 a day. All users write a star shape, namely a pentagram, during each trial, which all can achieve easily. Personal characteristics are also easily reflected in the star shape, making it a 
suitable pattern to evaluate malicious identification [6]. The following three methods, M1, M2, and M3, are defined for comparative evaluation as follows:

- M1 is the conventional method [4], whereby the enrollment template is not updated at all.

- M2 is the proposed method \#1, which simply updates the enrollment template for every identified trial.

- M3 is the proposed method \#2, which furnishes thresholds $T_{l}$ and $T_{h}$ to update the enrollment template adaptively.

\subsection{Tolerances to the Long Term Fluctuations by Update}

The transitions of False Rejection Rate: FRR were measured by M1 and M2 during the experimental period, where the threshold $T$ for personal identification is fixed to 0.32 and the same value of 0.5 was used for both weights $\boldsymbol{w}_{u}$ and $\boldsymbol{w}_{v}$ of the weighted average. FRR is the rate of erroneous cases when the valid user is incorrectly rejected. 12 persons had participated in this experiment and Figure 7 shows the average of their results. The horizontal axis indicates the trial date counted from the beginning of experiment, while the vertical axis indicates the FRR in all trials until that day.

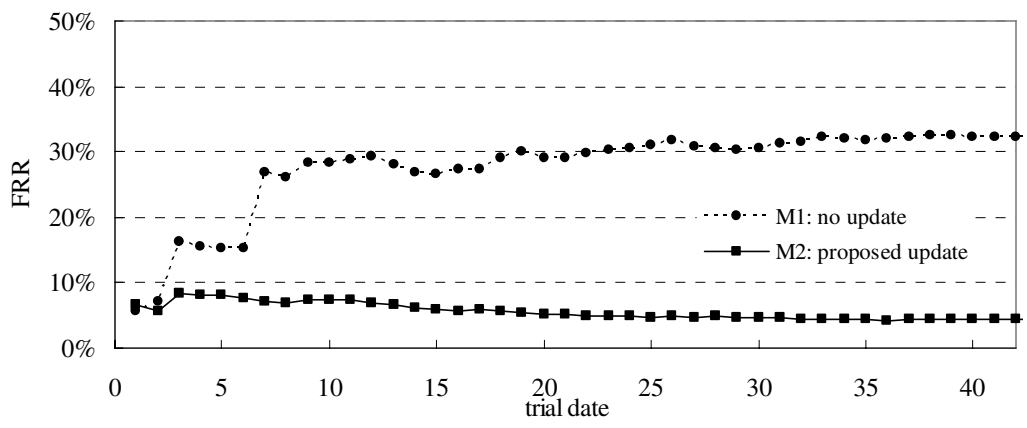

Fig. 7. Transition of verification accuracy over 6 weeks

Although the conventional method M1 maintains a low FRR of about $8 \%$ during the several initial days of the experiment, the FRR of M1 tends to rise over time; finally reaching $32.2 \%$. This is probably due to the fact that the first enrollment template does not go with the latest arm swing motion. On the contrary, the proposed method M2 maintains a lower FRR than M1 from the beginning of the experiment and also achieves a preferable downward curve through the experiment. This result confirms that long term stability is achieved in the arm swing identification by M2, where the enrollment templates are simply updated for every identified trial.

Figure 8 shows the False Rejection Rate: FRR and False Acceptance Rate: FAR curves of M1 and M2. An enlarged graph around the cross point is also exhibited in Figure 8, while the performances of the methods are comparatively evaluated in terms of the Equivalent Error Rate: EER [6], which is the percentage when the FRR is equal to the FAR. M1 has an EER of $14.7 \%$, while M2 achieves an EER of $4.7 \%$. The above results confirm that the proposed update method improves the verification 


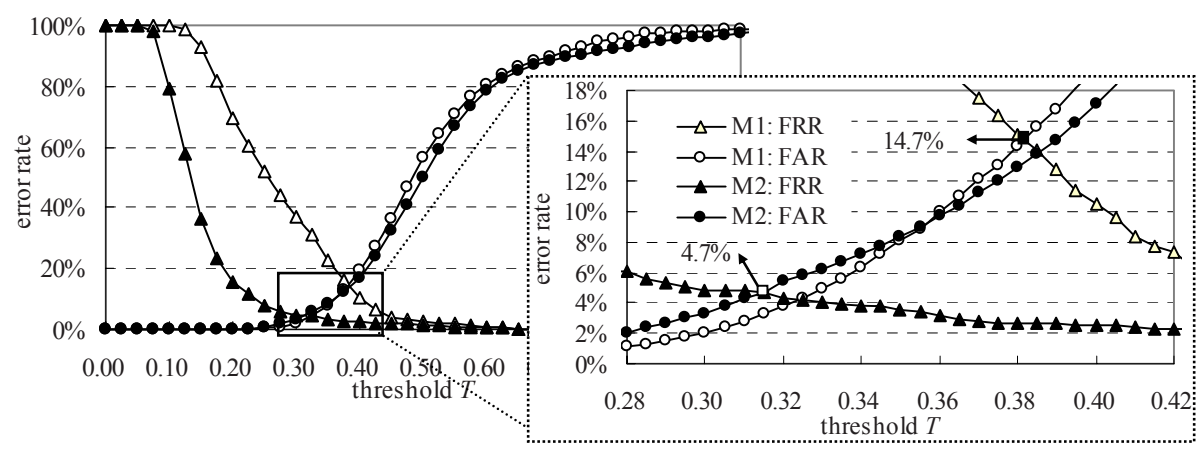

Fig. 8. FRR and FAR curve. The right graph shows an enlarged area around the cross point

accuracy of arm swing motion. However, Figure 8 also shows that FAR curves remain virtually unchanged between $M 1$ and $M 2$, although the FRR curve of M2 is considerably improved by updating. This is because the proposed update methods concentrate on reflecting the characteristics of the valid user so that new templates approach the center of distribution and the variance of his/her own probability distribution is reduced, as mentioned in the beginning of Section 3. Consequently, via the update, the rate of acceptance of the valid user increases and the FRR decreases, whereas FAR improvement is left for future work.

\subsection{Effectiveness of the Adaptive Update}

In this section, we investigate the effectiveness of M3, which has the adaptive update, as well as optimizing the thresholds $T_{l}$ and $T_{h}$ of M3. Table 1 shows certain pairs of thresholds $T_{l}$ and $T_{h}$, determined based on the probability interval of the distribution on differences. The table also shows the M3's EER and the update frequency, which represents the ratio of occurrence of the update, when the threshold pairs are used.

M3 works most effectively, when $T_{l}=0.095$ and $T_{h}=0.369$, corresponding to a $90 \%$ interval of the probability distribution. Under these conditions, M3 achieves the best EER of $4.11 \%$, which is improved by 0.6 points in comparison with the simple update method, M2. Furthermore, under these conditions, the update frequency of M3 is $83.2 \%$, which is lower than that of M2 by 16.8 points. These results confirm that M3 significantly maintains its template effectively and reduces the number of needless updates.

Table 1. Probability interval and threshold for adaptive update. $0 \%$ is equivalent to the conventional method M1 and 100\% is equivalent to the simple update method M2.

\begin{tabular}{l|c|c|c|c|c|c|c|c}
\hline Probability interval & $0 \%$ & $20 \%$ & $40 \%$ & $60 \%$ & $80 \%$ & $90 \%$ & $95 \%$ & $100 \%$ \\
\hline Threshold $T_{l}$ & -- & 0.142 & 0.136 & 0.125 & 0.110 & 0.095 & 0.082 & 0 \\
\hline Threshold $T_{h}$ & -- & 0.181 & 0.211 & 0.244 & 0.301 & 0.369 & 0.452 & $+\infty$ \\
\hline EER & 14.71 & 10.88 & 5.06 & 4.72 & 4.65 & 4.11 & 4.40 & 4.71 \\
\hline Cross point & 0.382 & 0.358 & 0.320 & 0.318 & 0.315 & 0.308 & 0.310 & 0.233 \\
\hline Update frequency & $0 \%$ & $16.0 \%$ & $34.3 \%$ & $49.3 \%$ & $68.0 \%$ & $\underline{83.2 \%}$ & $92.3 \%$ & $100 \%$ \\
\hline
\end{tabular}


We also conducted the leave-one-out cross validation test [7][8] for further assessment of the adaptive update method. The group of 12 persons is divided into 11 persons for training and one individual for evaluation respectively. The leave-one-out cross validation test checks whether the thresholds $T_{l}$ and $T_{h}$, determined from the 11 persons, are also effective for another individual not included in the group. There are 12 cases of group division and the performance of the method is estimated based on the average of results in all cases. The experimental result based on the leave-one-out cross validation test indicates an EER of $4.30 \%$, which falls below the optimized EER of $4.11 \%$ measured in section 4.2. Both the leave-one-out cross validation and optimized experiment indicate a relatively similar EER, with an accuracy reduction of no more than 0.19 points. These results lead to the following conclusions, namely that $T_{l}=0.095$ and $T_{h}=0.369$ prove to be the best thresholds in the adaptive update.

The EER performance of the proposed method is comparable to that of other identifications, based on human behaviors such as speech, signatures and gestures [1][3][9]. Their EER fall roughly 2 to $8 \%$ in the case of limited acquisition accuracy, for example, a small camera capturing images with insufficient illumination and so on, which represent typical circumstances facing mobile terminals.

\subsection{Analysis of the Weighting Parameter Characteristics}

Here, we also investigate the semi-optimal weights $w_{u}$ and $w_{v}$ for the weighted average. The thresholds in the adaptive update method M3 are fixed at $T_{l}=0.095$ and $T_{h}=0.369$, which achieves maximum efficiency in the previous experiment in section 4.2, while EER is also measured for each pair of weights $w_{u}$ and $w_{v}$ in Table 2. The pair of weights $w_{u}=0.6$ and $w_{v}=0.4$ achieves maximum efficiency, with an EER of $4.03 \%$ - results which lead to the following considerations. It is appropriate that the weighted average impose a slightly larger weight on the side of the enrollment template than that on the side of input motion. Appropriate weights prevent the original elements in the current enrollment template from being radically lost and also prevent the updated template from being degraded through accidentally input motion.

Table 2. Relations between the weighted average and EER

\begin{tabular}{l|c|c|c|c|c|c|c|c}
\hline Weights $\left(w_{u} / w_{v}\right)$ & $1.0 / 0.0$ & $0.8 / 0.2$ & $0.7 / 0.3$ & $0.6 / 0.4$ & $0.5 / 0.5$ & $0.4 / 0.6$ & $0.3 / 0.7$ & $0.0 / 1.0$ \\
\hline EER & 6.86 & 4.56 & 4.22 & $\underline{4.03}$ & 4.11 & 4.11 & 4.17 & 4.68 \\
\hline Cross point & 0.351 & 0.310 & 0.310 & 0.310 & 0.308 & 0.310 & 0.308 & 0.315 \\
\hline Update frequency & $94.5 \%$ & $83.9 \%$ & $82.4 \%$ & $\underline{82.2 \%}$ & $83.2 \%$ & $84.0 \%$ & $84.9 \%$ & $89.2 \%$ \\
\hline
\end{tabular}

\section{Conclusion}

This paper proposes a novel and stable human identification method based on arm swing motions. It also includes a common substantial problem, namely, an increase in the error rate over time like other human behavioral identifications. We introduce an adaptive update approach into the basic arm swing authentication method, and present a practical algorithm, using the DP-matching minimum route, to update the enrollment template. The algorithm can reflect a new tendency of motion while also preventing accidental movement from being learnt. Identification experiments show 
that EER decreases over time and reaches $4.0 \%$. These preferable results are surely confirmed by the leave-one-out method and this performance is comparable to that of other identification, based on human behaviors such as speech, signatures and gestures. Although the proposed method requires an additional process to update a template, its computational complexity is executable on cellular phones. Moreover, our authentication method can be significantly improved when the arm swing motion is not limited to the star shape. Allowing an arbitrary arm swing for each individual contributes to decreasing FAR, meaning we can achieve much lower EER in the practical reformation. Thus, our method can achieve high accuracy in terms of operational adjustment and is quite suitable for authentication in mobile terminals with natural behavior.

Acknowledgments. The authors would like to thank Dr. Shigeyuki Akiba, Dr. Shuichi Matsumoto, Dr. Yasuyuki Nakajima, Dr. Atsushi Koike, of KDDI R\&D Labs. and Dr. Akira Kubota of the Tokyo Institute of Technology, for their continuous support and encouragement.

\section{References}

1. Kato, T., Kawai, H.: A text-prompted speaker verification system implemented on a cellular phone and a mobile terminal. In: Proc. of ESCA ISCA, pp. 945-948 (2006)

2. Hangai, S., Yamanaka, S., Hamamoto, T.: On-line signature verification based on altitude and direction of pen movement. In: Proc. of IEEE ICME, vol. 1, pp. 489-492 (2000)

3. Patel, S.N., Pierce, J.S., Abowd, G.D.: A Gesture-based Authentication Scheme for Untrusted Public Terminal. In: Proc. of UIST, pp. 157-160 (2004)

4. Okumura, F., Kubota, A., Hatori, Y., Matsuo, K., Hashimoto, M., Koike, A.: A study on biometric identification based on arm sweep action with acceleration sensor. In: Proc. of IEEE ISPACS, pp. 219-222 (2006)

5. Uludag, U., Ross, A., Jain, A.K.: Biometric template selection and update: case study in fingerprints. Pattern Recognition 37(7), 1533-1542 (2004)

6. Mansfield, A.J., Wayman, J.L.: Best practices in testing and reporting performance of biometric devices version 2.01. NPL Report CMSC 14/02 (August 2002)

7. Shahraray, B., Anderson, D.J.: Optimal estimation of contour properties by cross-validated regularization. IEEE Trans. on PAMI 11(6), 600-610 (1989)

8. Krzanowski, W.J.: Cross-validation in principal component analysis. Biometrics 43, 575584 (1987)

9. Jain, A.K., Bolle, R., Pankanti, S.: Biometrics: Personal identification in network society. Kluwer Academic Publishers, Dordrecht (1999) 